\title{
Response latencies to the onset and offset of visual stimuli
}

\author{
VINCENT DI LOLLO and JAMES T. ENNS \\ University of British Columbia, Vancouver, British Columbia, Canada \\ STEVE YANTIS \\ Johns Hopkins University, Baltimore, Maryland \\ and \\ LYNDA G. DECHIEF \\ University of British Columbia, Vancouver, British Columbia, Canada
}

\begin{abstract}
Simple response times (RTs) are known to be slower to the offset of a visual stimulus than to its onset. This is called the onset advantage. In the first of four experiments, we discovered that a spurious onset advantage can be produced by the long persistence of P31 phosphor. In the remaining three experiments, we found that offset RTs were slower only when they were made in a context in which responses to the abrupt onset of some stimuli had to be suppressed. We tested this hypothesis of response suppression in two ways: (1) by mixing regular onset trials with other trials on which a response to an onset had to be suppressed, and (2) by ramping the emergence of "offset" stimuli over time, so that offsets were the only abrupt events in the display. In both cases, we found that the onset advantage depended critically on whether the responses were made in a context of response suppression. We conclude that the onset advantage is mediated not by sensory factors such as visible persistence, but by response programming factors that are strongly affected by contextual events.
\end{abstract}

Response times (RTs) are known to be faster to the sudden onset of a visual stimulus than to its offset. This onset advantage has been found reliably in different populations of observers and under diverse viewing conditions (Baro, Brzezicki, Lehmkuhle, \& Hughes, 1992; Breitmeyer, Levi, \& Harwerth, 1981; Briggs \& Kinsbourne, 1972; May, Martin, MacCana, \& Lovegrove, 1988; Parker, 1980; Smith, Early, \& Grogan, 1986). A common account of the onset advantage, first proposed by Briggs and Kinsbourne (1972), is based on visible persistence, which refers to a brief period of continued visibility after the physical stimulus has been turned off (Coltheart, 1980). Visible persistence is thought to produce an onset advantage indirectly by extending the phenomenal duration of the stimulus. The reasoning is as follows. A response to either the onset or the offset of a stimulus is initiated as soon as a critical change in intensity has been perceived. In the case of an onset, a response is made as soon as a critical increment in intensity is perceived. This occurs promptly, as soon as the stimulus is turned on. In the case of an offset, a response is

This work was supported by research grants from the Natural Sciences and Engineering Research Council of Canada to V.D.L. and to J.T.E., from the National Institute of Mental Health (R01-MH43924) to S.Y., and by a NATO travel grant (CRG 960714) to V.D.L., J.T.E., and S.Y. Correspondence should be addressed to V. Di Lollo, Department of Psychology, University of British Columbia, 2136 West Mall, Vancouver, BC, V6T 1 Z4 Canada (e-mail: enzo@interchange.ubc.ca). made as soon as a critical decrement in intensity is perceived. This, however, does not occur as promptly because visible persistence delays the perception of the drop in intensity.

Despite its appealing simplicity, this account is questionable on at least two grounds. First, in earlier investigations, the stimuli were displayed for relatively long durations, often $500 \mathrm{msec}$ or longer. This is an important consideration because the duration of visible persistence is inversely related to the duration of the inducing stimulus (inverse-duration effect; Di Lollo, 1980; Efron, 1970). Notably, at exposure durations beyond about $100 \mathrm{msec}$, stimuli produce little, if any, visible persistence. It seems implausible, therefore, that an onset advantage as large as $200 \mathrm{msec}$ could be based on visible persistence alone (see, e.g., Breitmeyer et al., 1981). Second, when specified in the earlier published reports, the screen phosphor used was invariably P31. The luminescence of P31 phosphor is known to remain visible for periods in excess of $1 \mathrm{sec}$, even when the screen is illuminated with a veiling light (Di Lollo, Seiffert, Burchett, Rabeeh, \& Ruman, 1997). The phosphor's visibility is further enhanced if the viewing is done under dim-lighting or dark-adapted conditions, which was the case in earlier studies of the onset advantage.

On the strength of this evidence, visible persistence is called into question as an exclusive basis for the onset advantage. Rather, we should consider the extent to which the onset advantage might stem not from visible persis- 
tence within the observer's visual system but from phosphor persistence on the display screen. We investigated this issue by recording RTs to onsets and offsets of stimuli displayed on three different screens: an oscilloscope equipped with P31 phosphor; another oscilloscope equipped with $\mathrm{P} 15$ phosphor, known to be free from persistence (Di Lollo et al., 1997); and an AppleVision computer monitor. We expected that, to the extent that phosphor persistence mediated the onset advantage in the earlier studies, we would obtain an onset advantage with P31 phosphor but not with P15 phosphor. The AppleVision screen was included for purposes of comparison.

The phosphor-persistence hypothesis was clearly supported in the first of four experiments. Further, the results hinted at an additional factor, hitherto unsuspected but of potential theoretical significance, capable of influencing the onset advantage. The new factor appeared to be related to the sequencing of onset and offset trials within an experimental session. This was confirmed in the second experiment, in which an onset advantage was obtained when onset and offset trials were presented in separate blocks (as was done in all earlier studies), but not when the two types of trials were intermingled randomly. These differences were investigated in two further experiments that strongly implicated response-set interference as a prime factor in the onset advantage.

\section{EXPERIMENT 1}

\section{Method}

Observers. Two of the authors and 29 undergraduate students at the University of British Columbia, who received course credit for their participation, acted as observers. All had normal or correctedto-normal vision. All displays were viewed binocularly with natural pupils.

Apparatus and Stimuli. Vertical square-wave gratings were displayed on three separate screens: two Tektronix 608 oscilloscopes, one equipped with P15 phosphor, the other with P31 phosphor, and an AppleVision 1710 display monitor driven by a Power Macintosh computer. A vertical square-wave grating, presented within a $2 \mathrm{deg} \times 2 \mathrm{deg}$ square area in the center of the screen, had spatial frequency of $12 \mathrm{c} / \mathrm{deg}$ as viewed from a distance of $57 \mathrm{~cm}$. The luminance of the light bars in each grating was $66 \mathrm{~cd} / \mathrm{m}^{2}$, and the dark bars had the same luminance as the background, which was $1 \mathrm{~cd} / \mathrm{m} 2$ for the AppleVision screen and $<.02 \mathrm{~cd} / \mathrm{m}^{2}$ for the two oscilloscopes. Observers sat facing the screen and entered their responses on a computer keyboard illuminated by a $15-\mathrm{W}$ shielded fluorescent bulb that provided the only illumination in the room.

Procedure. After 20 practice trials, observers were presented with 100 trials on each of the three display screens, with order of display counterbalanced across observers. Onset and offset trials were sequenced randomly to counter effects of order. Onset trials began with the word ON displayed 2 deg above the center of the screen. The word subtended 2 deg of visual angle, and was displayed for $2 \mathrm{sec}$. After the word disappeared, the screen remained blank for a variable period of $1,500,1,750,2,000,2,250$, or $2,500 \mathrm{msec}$. The grating was then displayed in the center of the screen, and it remained on view until the observer responded. Offset trials began with a simultaneous display of the word OFF and the grating in the same locations as in the onset trials. After $2 \mathrm{sec}$, the word disappeared, and after a variable period (as in the onset trials), the grating disappeared. Observers were instructed to respond as quickly as possible to the onset of the grating in the trials labeled "ON," and to the offset of the grating in the trials labeled "OFF," by depressing the space bar on a keyboard. Each trial was terminated by the observer's response. The next trial began after a 2 -sec intertrial interval.

\section{Results and Discussion}

In Experiment 1 and in all the following experiments, RTs shorter than 100 or longer than 1,000 msec were discarded as representing the effects of anticipation or inattention. In every case, discarded responses accounted for less than $5 \%$ of all responses. Mean onset and offset RTs, averaged over all 31 observers, separately for the three screens, are shown in Figure 1. The corresponding frequency distributions are shown in Figure 2.

The results were analyzed in a 2 (response types: onset vs. offset) $\times 3$ (screen types: P15, P31, MAC) withinsubjects analysis of variance (ANOVA). The only significant effect revealed in the analysis was the interaction between screen type and response type $[F(2,20)=6.59$, $\left.M S_{e}=406.97, p<.003\right]$. Orthogonal comparisons (Fisher's $L S D$ ) revealed a significant onset advantage for the P31 screen $\left[F(1,20)=13.80, M S_{\mathrm{e}}=406.97, p<.001\right]$ but not for the P15 screen $\left[F(1,20)=3.73, M S_{\mathrm{e}}=406.97\right.$, $p>.05]$ or for the AppleVision screen $[F(1,20)=1.81$, $\left.M S_{\mathrm{e}}=406.97, p>.05\right]$.

The hypothesis that phosphor persistence might have played a part in earlier studies of the onset advantage is supported by the data. Slower responding to the offset of a stimulus than to its onset is attributable to a persisting image of the stimulus on the screen. This is true for screens equipped with P31 phosphor, but not for those equipped with P15 phosphor or for AppleVision screens.

We hasten to add that this finding does not constitute conclusive evidence that all earlier studies were vitiated by a confounding with phosphor persistence. Indeed, some studies (e.g., Smith et al., 1986) revealed a significant onset advantage with phosphors other than P31.

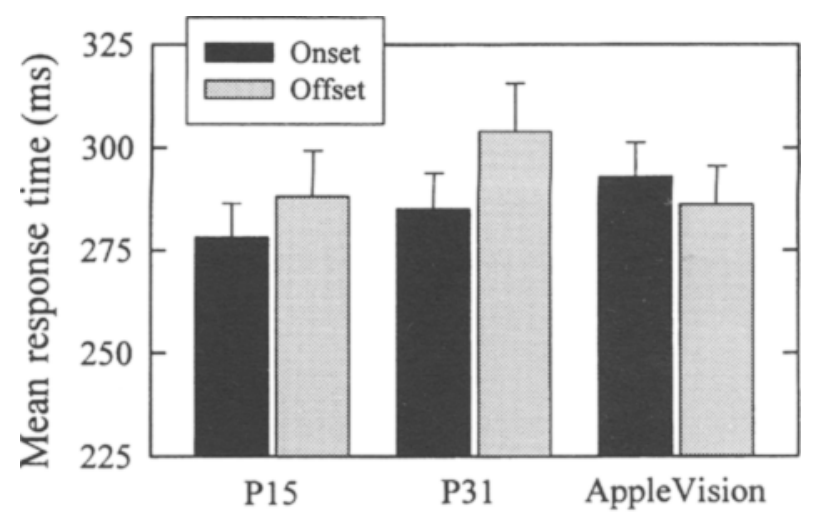

Screen

Figure 1. Experiment 1. Response times to stimulus onset and offset, averaged over all observers, separately for each of the three display screens. 


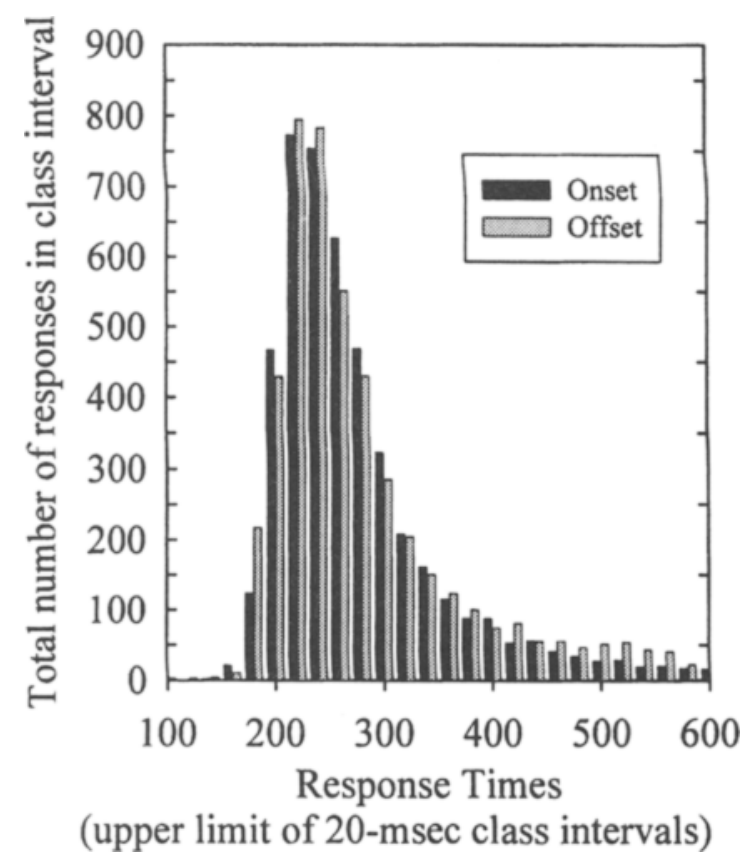

Figure 2. Experiment 1. Frequency distribution of response times to stimulus onset and offset, combined for all observers and display screens.

This underscores a surprising outcome illustrated in Figure 1. Namely, no significant onset advantage was obtained in the present study, either with $\mathrm{P} 15$ phosphor or with the AppleVision screen.

In examining possible reasons why an onset advantage was found in earlier studies that were free of phosphor confounding (e.g., Smith et al., 1986) but not in the present study, we undertook a systematic comparison between our procedures and the procedures employed in earlier studies. We began by reasoning that the onset advantage revealed in those studies was unlikely to have stemmed from visible persistence. Given the long exposure durations employed in the offset conditions in all extant experiments, including the present one, the inverse-duration effect would militate against a persistence account.

Instead, the most notable difference between the procedures employed in the present and in earlier experiments lies in the sequence in which the onset and offset trials were presented within an experimental session. In the present experiment, onset and offset trials were sequenced randomly. In contrast, in all experiments that reported an onset advantage, onset and offset trials were presented in separate blocks. Sequencing differences such as these have been known to yield substantial differences in the experimental outcomes, possibly because of differences in response set (see Eriksen \& Eriksen, 1974). Experiment 2 was designed to find out whether the onset advantage is affected by the sequencing of onset and offset trials within an experimental session.

\section{EXPERIMENT 2}

In conventional studies of the onset advantage, onset trials and offset trials have always been presented in separate blocks. In Experiment 1, the two types of trials were mixed randomly within a single block, and the onset advantage failed to appear. In the present experiment, the sequencing of onset and offset trials was varied explicitly. In one condition, the two types of trials were presented in separate blocks, as in earlier studies. In another condition, they were mixed randomly, as in Experiment 1 . To the extent that sequencing is an important determinant, we expected to find an onset advantage when onset and offset trials were blocked, but not when they were mixed.

\section{Method}

Observers. The observers were two of the authors and 20 naive undergraduate students at the University of British Columbia who received course credit for participating. All had normal or correctedto-normal vision. All displays were viewed binocularly with natural pupils.

Apparatus and Stimuli. Vertical square-wave gratings were displayed within a 2 deg $\times 2$ deg square area in the center of an AppleVision 1710 display monitor. The gratings had spatial frequency of $12 \mathrm{c} / \mathrm{deg}$ as viewed from a distance of $57 \mathrm{~cm}$. The luminance of the light bars in each grating was $66 \mathrm{~cd} / \mathrm{m}^{2}$, and that of the dark bars was $1 \mathrm{~cd} / \mathrm{m} 2$. The luminance of the background was $32 \mathrm{~cd} / \mathrm{m}^{2}$. Observers sat facing the screen and entered their responses on a computer keyboard. The room was dimly lit by attenuated overhead fluorescent bulbs.

Procedure. Procedures were the same as in Experiment 1, with the following exceptions. Onset and offset trials were presented as either blocked or mixed. The blocked conditions consisted of two sets of 150 trials. One set contained only onset trials, the other only offset trials. The mixed condition consisted of 150 onset trials and 150 offset trials, sequenced randomly, for a total of 300 trials. One half of the participants ( 11 observers) did the onset trials before the offset trials. The sequence was reversed for the remaining $11 \mathrm{ob}-$ servers. This was followed with 300 trials of the mixed condition. Suitable rest periods were introduced at the end of every 150 trials. The timing and the appearance of the displays were the same as in Experiment 1, including the word ON or OFF, which was displayed on each trial, even in the blocked condition.

\section{Results and Discussion}

Mean onset and offset RTs in Experiment 2, averaged over all 22 observers, are shown in Figure 3, separately for blocked and mixed trial sequences. The results were analyzed in a 2 (response type: onset vs. offset) $\times 2$ (trial sequence: blocked vs. mixed) $\times 2$ (sequence order: onset first vs. offset first) within-subjects ANOVA. The only significant effect revealed in the analysis was the interaction effect between response type and trial sequence $\left[F(1,20)=5.92, M S_{\mathrm{e}}=315.25, p<.05\right]$. The other nonsignificant effects were as follows: response type $[F(1,20)$ $\left.=3.90, M S_{\mathrm{e}}=634.65, p>.05\right]$, trial sequence $[F(1,20)=$ $\left.2.61, M S_{\mathrm{e}}=484.93, p>.05\right]$, sequence order $[F(1,20)<$ $1]$, and response type $\times$ trial sequence $\times$ sequence order interaction $[F(1,20)<1]$. 


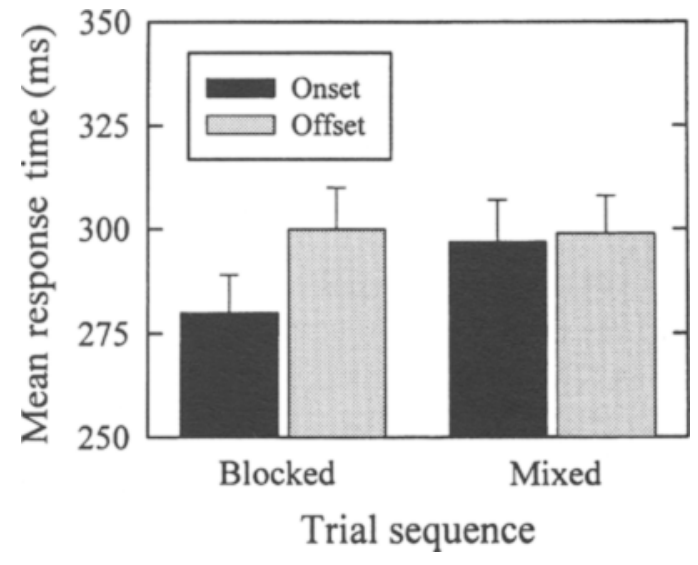

Figure 3. Experiment 2. Mean response times to stimulus onset and offset, separately for blocked and mixed trial sequences. In the blocked condition, any given block contained either onset or offset trials. In the mixed condition, onset and offset trials were sequenced randomly within a block.

The significant interaction effect between response type and trial sequence confirmed the graphical evidence in Figure 3 that an onset advantage occurred when onset and offset trials were presented in separate blocks but not when they were mixed. By presenting onset and offset trials in separate blocks, as in all earlier studies, we replicated the onset advantage conventionally reported in the literature. Also, as in Experiment 1, we failed to find an onset advantage when the trials were mixed and the stimuli were presented on a screen (AppleVision monitor) with low phosphor persistence.

This pattern of results is inconsistent with an account based on visible persistence alone. If mixing of trials can eliminate the difference between onset and offset RTs, the explanation that visible persistence is responsible for producing the onset advantage is called into question. This is so because, considered in isolation, any given offset trial was exactly the same as any other offset trial, whether it occurred in an homogeneous or in a mixed context. It was also the case that successive trials were separated in time by several seconds, during which stimulusbound effects such as receptor adaptation and transient effects of stimulation would have abated. It is implausible, therefore, that the duration of visible persistence on any given trial was affected by the response made on preceding trials. At any rate, sequential dependencies were ruled out by a further analysis in which offset trials were grouped separately, depending on whether they were preceded by onset or offset trials. No sequential dependencies were revealed in this analysis: Offset RTs were unaffected by the type or number of preceding trials.

From the results of the sequential analysis, it is clear that whatever factors were responsible for the absence of the onset advantage in the mixed condition remained relatively stable throughout a session, as opposed to changing from trial to trial. As a first step in identifying the key factors, we considered whether mixing onset and offset trials led to faster offset RTs or to slower onset RTs than did the blocked presentation. The latter option is clearly supported by the data in Figure 3. Offset RTs remained about the same regardless of context, but onset RTs were slower in the mixed condition. On the face of it, something inherent in the mixing of trials led to a slowdown in the onset RTs.

Pursuing this line of reasoning, it becomes plausible that, in the mixed condition, observers faced a conflict in making appropriate responses on onset and offset trials. This is so because, on any given trial, the sudden appearance of the grating was a signal for two incompatible responses. On onset trials, the sudden appearance of the grating was a signal to make a speeded response. In contrast, on offset trials, the onset of the grating was a signal to withhold the response until the grating disappeared. Therefore, any tendency to respond to the abrupt onset of the grating on offset trials had to be suppressed. The contingency in which a given signal is associated with two incompatible responses has been studied extensively, and is known to produce negative transfer (Osgood, 1953, chap. 12). From this perspective, it is plausible that the act of suppressing the response to stimulus onset on offset trials might have interfered with the speeded response to stimulus onset on onset trials. It should be noted that observers were never required to withhold responses to the offset of the grating. Therefore, a corresponding response conflict involving offset RTs could not occur. This hypothesis of generalized response suppression was examined in Experiment 3.

\section{EXPERIMENT 3}

Experiment 3 was designed to examine whether onset RTs are slowed down by presenting the stimuli within a "response suppression" context. Such a context was again provided by a mixing of trials, but instead of mixing onsets with offsets, we mixed simple onset trials with other onset trials on which observers were required to withhold the response. This was done by mixing onset trials with "second-onset" trials. In second-onset trials, two gratings were presented in sequence, and observers were instructed to withhold their response to the first stimulus and to respond only to the onset of the second. If the context of response suppression acts to slow response times, there should be slower RTs with this mixing.

In a control condition, a separate group of observers was presented with precisely the same sequence, but was instructed to respond to the onset of every stimulus, whether it came first or second. This condition was identical to the experimental condition in every respect, except for the withholding of the response on second-onset trials.

\section{Method}

Observers. The observers were 36 naive undergraduate students at the University of British Columbia who received course credit 


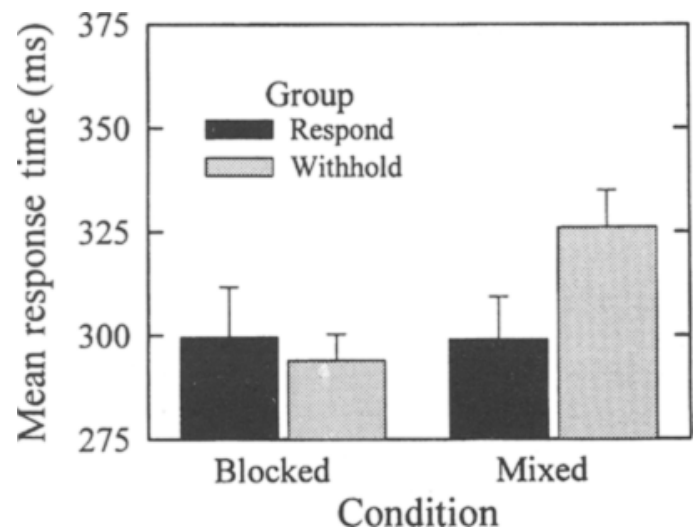

Figure 4. Experiment 3. Mean response times in the respond and withhold conditions, separately for blocked and mixed trial sequences. See text for explanation.

for participating. All had normal or corrected-to-normal vision. All displays were viewed binocularly with natural pupils.

Stimuli and Procedure. Stimuli and procedures were the same as in Experiment 2, except for the following. Experiment 3 contained only onset trials. The 36 participants were assigned to two groups of 18 observers each. In the experimental group, the withhold group, observers were required to withhold their responses some of the time. In the control group, the respond group, observers always responded to all the stimuli. Each group served in two conditions: blocked and mixed. For the withhold group, blocked and mixed conditions were as follows. The blocked condition consisted of 100 trials identical to the blocked-onset condition in Experiment 2 . The mixed condition had two types of trials: single and second. Each single trial began with the word $O N, 2$ deg above the center of the screen, and continued with the presentation of the grating to which the observer responded, as was done in Experiment 2. Second trials involved the presentation of two gratings in sequence. Each trial began with the word SECOND displayed for $2 \mathrm{sec}, 2 \mathrm{deg}$ above the center of the screen. This was followed by a blank screen for $2 \mathrm{sec}$. Next, a grating appeared in one of two locations, $2 \mathrm{deg}$ to the left or to the right of center. After a variable period $(1,500$ 2,500 msec, as in Experiment 2), a second grating appeared in the empty location. Observers were instructed to respond only to the second of the two onsets. One hundred single trials were randomly interspersed with 100 second trials, for a total of 200 trials in the mixed condition. Nine observers did the blocked condition first, and the other 9 did the mixed condition first. For the respond group, the blocked and mixed conditions were exactly the same as for the withhold group, except that observers were instructed to respond to the onsets of both gratings on second trials. Therefore, observers in the respond group were never required to withhold their responses to the onset of any grating.

\section{Results and Discussion}

The data for analysis consisted of the RTs recorded on all single trials in the mixed conditions, and on the corresponding trials in the blocked condition. RTs for second trials in the mixed conditions were recorded but were not analyzed. Thus, each observer had two scores: one was the mean RT obtained by averaging the 100 responses to the onset of the grating in the single trials in the mixed condition. and the other was the mean RT obtained by averaging the 100 responses to the onset of the grating in the corresponding trials in the blocked condition.

An ANOVA was performed on these data, with one within-subjects factor (blocking: blocked trials vs. mixed trials) and two between-subjects factors (context: withholding vs. responding, and order: blocked first vs. mixed first). Only two effects were significant: blocking $[F(1,32)$ $\left.=9.45, M S_{\mathrm{e}}=508.61, p<.005\right]$, and the interaction effect between blocking and context $\left[F(1,32)=9.52, M S_{\mathrm{e}}=\right.$ $508.61, p<.005]$. The other nonsignificant effects were as follows: context $[F(1,32)<1]$, order $[F(1,32)<1]$, context $\times$ order $\left[F(1,32)=1.58, M S_{\mathrm{e}}=2866.67, p>\right.$ $.05]$, blocking $\times$ order $\left[F(1,32)=1.28, M S_{\mathrm{e}}=498.69\right.$, $p>.05]$, and blocking $\times$ context $\times$ order $[F(1,32)<1]$. The results, averaged over observers and orders, are shown in Figure 4, separately for blocked and mixed trials, and for the two contexts (withholding and responding).

Response times were approximately the same in the two blocked conditions (Figure 4). This was to be expected because, when trials were blocked, context was only a dummy variable. Namely, the two blocked conditions were identical, and neither was presented in a context of response suppression. On the other hand, context had a substantial effect on performance in the mixed conditions. RTs made in a context of response suppression (Figure 4, mixed withhold condition) were significantly slower than RTs made in a context in which response suppression never occurred (Figure 4, mixed respond condition).

There is an apparent inconsistency between the present results and those of Experiment 2. In Experiment 2 (Figure 3), RTs differed in the two blocked conditions but not in the two mixed conditions. In the present experiment, it was the other way around (Figure 4). A moment's reflection will show that, in fact, the critical slowdown in RT occurred only in the context of response suppression in the mixed condition in both experiments. In Experiment 2, suppression arose implicitly from having to withhold the response to stimulus onset on offset trials in the mixed condition. We have suggested that such suppression might have generalized to other onset responses in the mixed condition and was thus responsible for slower onset RTs as compared with the corresponding blocked condition (Figure 3 ). In the present experiment, suppression stemmed from withholding the response on second trials in the mixed condition. This was responsible for slower RTs in the mixed withhold condition as compared with the corresponding blocked condition (Figure 4). Thus, presenting onset trials within a "response-suppression" context, as in the present experiment, yields results homologous to those obtained when onset trials are presented in a context of offset trials, as in Experiment 2.

While not providing definitive proof of common causation, the parallel between the two sets of results is compelling. Suppressing the response to the onset of one stimulus can slow down the response to the onsets of an- 
other stimulus presented in the same context. In this sense, the two patterns of performance seen in Figures 3 and 4 can be regarded as different expressions of the same phenomenon.

Next, we ask whether the response suppression illustrated in the present experiment can account for the onset advantage seen with blocked presentations in Experiment 2 (Figure 3 ) and in previous studies. The answer is that it certainly can, when one considers that not only do a blocked onset trial and a blocked offset trial differ in the visual signal governing the response (onset and offset, respectively), but also that the offset signal on offset trials is always preceded by an onset signal that is to be ignored. No similar transient in the stimulus precedes the onset signal on onset trials. That is, in offset trials, the observer must, by instruction, suppress responding to a first abrupt event (stimulus onset) while making a speeded response to a second abrupt event (stimulus offset). To the extent that suppression generalizes from one abrupt event to the other, the speeded response to stimulus offset will be suppressed. More important, no such generalized suppression would occur on a corresponding onset trial (provided that trials are blocked) because no suppression of responses to stimulus offset is ever required, implicitly or explicitly, on onset trials. The upshot is an onset advantage. Experiment 4 is a test of this hypothesis.

\section{EXPERIMENT 4}

Experiment 4 was designed to examine whether withholding the response to the abrupt onset of a stimulus can generalize to - and, therefore, slow down-the response to the abrupt offset of that stimulus. In the present experiment, we compared offset RTs in two independent offset conditions that differed in respect to the mode of onset of the stimuli. The abrupt condition was the same as the blocked offset condition in Experiment 2. Each stimulus appeared abruptly on the screen, and observers responded to its offset. In the ramped condition, the stimulus appeared on the screen gradually. The grating began by being barely visible, and its intensity increased progressively to a maximum over a period of $1 \mathrm{sec}$. Given the gradualness of the onset, observers in the ramped condition never had to withhold a response to an abrupt stimulus. We reasoned that, to the extent that suppression of the response to abrupt onsets generalizes to abrupt offsets, offset RTs should be slower in the abrupt than in the ramped condition. A third condition, in which responses were made to abrupt onsets, was included to permit an assessment of the onset advantage.

\section{Method}

Observers. The observers were 45 naive undergraduate students at the University of British Columbia who received course credit for participating. All had normal or corrected-to-normal vision.

Stimuli and Procedure. Stimuli and procedures were the same as in Experiment 2, except for the following. All stimuli were displayed on a Tektronix 608 oscilloscope equipped with P15 phos- phor, which allowed finer control of the ramping than did the AppleVision monitor. The 45 participants were assigned randomily to three groups of 15 observers each. For the onset group, the stimulus sequence was the same as in the blocked onset condition in Experiment 2, with observers responding to the onset of each stimulus. For the abrupt group, the stimulus sequence was the same as in the blocked offset condition in Experiment 2, with observers responding to the offset of each stimulus. The ramped group received the same treatment as the abrupt group except that the grating appeared on the screen gradually over a period of $1.1 \mathrm{sec}$. The luminance of the bright bars of the grating started at $3 \mathrm{~cd} / \mathrm{m}^{2}$ and increased by $3 \mathrm{~cd} / \mathrm{m}^{2}$ every $50 \mathrm{msec}$ to a maximum of $66 \mathrm{~cd} / \mathrm{m}^{2}$, which matched the luminance of the gratings in the other two groups. The grating continued to be displayed at the maximum luminance for an additional period that varied randomly between 2,500 and $3,500 \mathrm{msec}$, in steps of $250 \mathrm{msec}$, and was then turned off abruptly. Observers responded to the offset of the grating by pressing the space bar. All three groups received 20 practice trials followed by 100 experimental trials.

\section{Results and Discussion}

Mean RTs are shown in Figure 5, separately for the three groups. The results were analyzed in a betweensubjects ANOVA, with groups as the only factor. The analysis revealed a significant difference among the groups $\left[F(2,42)=5.55, M S_{\mathrm{e}}=1,516.10, p<.01\right]$. Individual tests of significance (Fisher's Protected LSD) showed that mean RTs in the abrupt group were significantly slower than those in either the onset group $\left[F(1,42)=9.90, M S_{\mathrm{e}}=1,516.10, p<.01\right]$ or the ramped group $\left[F(1,42)=6.36, M S_{\mathrm{e}}=1,516.10, p<.02\right]$. The latter two groups did not differ significantly from each other $[F(1,42)<1]$.

By comparing the onset group with each of the two offset groups, it is possible to obtain two estimates of the onset advantage. Comparison between the onset group

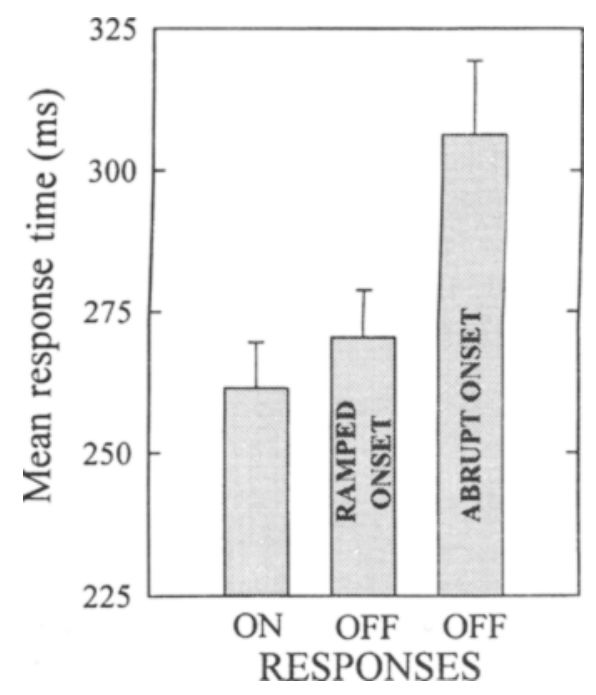

Figure 5. Experiment 4. Mean response times to stimulus onset (left bar), to stimulus offset when the stimuli had ramped onsets (central bar), and to stimulus offsets when the stimuli had abrupt onset (right bar). The three bars illustrate the results of three separate groups of observers. 
and the offset group that experienced abrupt onsets reveals a conventional onset advantage similar to that found in the blocked conditions of Experiment 2. In contrast, no onset advantage is revealed by a comparison with the offset group that experienced ramped onsets.

This pattern of results supports the hypothesis that withholding the response to an abrupt onset leads to suppression of the response to other abrupt stimuli, notably abrupt offsets. In the present experiment, this led to slower RTs in the offset group that experienced abrupt onsets. No such suppression could occur in the offset group that experienced ramped onsets because observers never had to withhold responses to abrupt stimuli. Thus, an onset advantage is seen when the onset group is compared with the abrupt group, but not when it is compared with the ramped group.

\section{GENERAL DISCUSSION}

A commonly held view of the onset advantage is that it occurs because the perception of stimulus offset is delayed by the duration of visible persistence (e.g., Briggs $\&$ Kinsbourne, 1972). The outcome of the present work does not support that view. In the course of investigating phosphor persistence as a possible confounding factor in earlier experiments (Experiment 1), we discovered that the onset advantage fails to appear when onset and offset trials are intermixed instead of being presented in separate blocks, as had been done in all previous studies. This led to an examination of response-context effects. Experiments 2 and 3 revealed that when the context requires suppression of the response to the onset of some stimuli, this suppression generalizes to other onset responses. When this occurs, responses to stimulus onset are slowed down to the level of offset responses, thereby abolishing the onset advantage. This is illustrated in Figure 3 where the onset advantage is very much in evidence in the blocked condition but not in the mixed condition. In Experiment 4 we examined and confirmed the hypothesis that suppressing the response to abrupt onsets generalizes not only to other onsets but also to other abrupt stimuli, notably abrupt offsets. This implicates response suppression as the main factor underlying the onset advantage, whether stimulus presentation is mixed or blocked.

Collectively, these results strongly suggest that the onset advantage is mediated not by sensory factors that affect the perception of onsets and offsets differently, but by response programming factors that are strongly affected by contextual events. More specifically, the onset advantage occurs not because onsets are perceived more rapidly than offsets, but because offsets RTs are slowed down by dint of being made in a context in which responses to abrupt onsets must be suppressed.

The present results leave little doubt that responseprogramming factors play a major role in the onset advantage. But this does not mean that low-level sensory factors such as visible persistence may not also contribute, at least in some measure. We now examine what contributions, if any, low-level factors may make to the onset advantage.

The earliest processing level worth considering is that of the receptors. It is conceivable that an onset advantage could be mediated by receptor adaptation as follows. Receptors may respond readily to stimulus onset, but more sluggishly to stimulus offset, because responsiveness is lowered through adaptation during the period that the stimulus is on display. Receptor adaptation, however, can be readily dismissed as a potential contributing factor. It is known that the perceptual latency to the offset of a brief stimulus is inversely related to stimulus duration (e.g., Efron, 1970). This is the opposite of what might be expected on the basis of receptor adaptation. Besides, there is no cogent reason why adaptation should be affected differently by the type of contextual manipulations implemented in Experiments 2, 3, and 4.

One possible exception to the involvement of low-level factors in the onset advantage should be noted. In a study of the effect of flicker masking on temporal sensitivity over a range of spatial frequencies, Breitmeyer et al. (1981) reported finding an onset advantage when the spatial frequency of the stimulus was high, but not when it was low. This must be regarded as evidence of a possible involvement of low-level sensory mechanisms in the onset advantage. But the evidence on this issue is far from uniform. Two studies designed explicitly for this purpose showed the onset advantage to be the same at all spatial frequencies tested (Baro et al., 1992; Parker, 1980). The results of a third study were inconclusive (Smith et al., 1986). To be sure, an overall onset advantage, independent of spatial frequency, was obtained in each of these studies. In every case, however, onsets and offsets were tested in separate blocks of trials, and all stimuli had abrupt onsets. The ensuing onset advantage, therefore, can be explained readily by the response-suppression effects illustrated in the present study.

Further evidence inconsistent with low-level sensory involvement is supplied by event-related potentials of brief visual stimuli. The latencies of the electrophysiological $\mathrm{ON}$ and OFF responses triggered by stimulus onset and offset are especially relevant to the visiblepersistence hypothesis of the onset advantage. To reiterate, this hypothesis stipulates that whereas the onset of a stimulus is perceived promptly, perception of stimulus offset is delayed by visible persistence. More precisely, the onset advantage is said to stem from latency differences in the sensory registration of onset and offset signals. If so, one should see corresponding differences in the latency of brain responses triggered by the onsets and offsets of appropriate visual stimuli.

Precisely this type of information was gathered by Servière, Miceli, and Galifret (1977). Visual evoked potentials triggered by stimuli ranging from 0.5 to $500 \mathrm{msec}$ were recorded from occipital sites. To compensate for 
time-intensity reciprocity (Bloch's law), stimuli were displayed at a constant perceived intensity across all exposure durations. Latencies of the $\mathrm{P}_{1}$-on and $\mathrm{P}_{1}$-off components reached asymptote at an exposure duration of about $50 \mathrm{msec}$, and remained stable at all longer durations. According to the persistence hypothesis, longer latencies should be expected for offsets than for onsets because of visible persistence. In fact, the results revealed the opposite pattern. Asymptotic $\mathrm{P}_{1}$-off latencies were actually shorter $(73 \mathrm{msec})$ than the corresponding $\mathrm{P}_{1}$-on latencies $(93 \mathrm{msec})$. This is entirely in keeping with the present view that the onset advantage stems not from sensory events in early vision but from response programming that takes place later in the chain of processing events.

Visible persistence becomes even more questionable as an explanatory principle if one considers the inverseduration effect. With the single exception of an experiment by Baro et al. (1992) in which the duration of the stimuli ranged from 50 to $400 \mathrm{msec}$, studies of the onset advantage have employed exposure durations ranging from 0.5 to several seconds. This is understandable in that long exposure durations are necessary to decouple onset from offset responses. At the same time, however, long exposure durations render a persistence account less credible because stimuli longer than about $100 \mathrm{msec}$ are known to yield little, if any, visible persistence (Coltheart, 1980; Di Lollo, 1980; Efron, 1970).

Low-level factors seem to be all but ruled out by this evidence. In contrast, the response-suppression account advocated here can account for the present results and for the bulk of the results in the experimental literature.

\section{REFERENCES}

Baro, J. A., Brzezicki, L. J., Lehmkuhle, S., \& Hughes, H. C. (1992). The perceived duration of gratings. Perception, 21, 161-166.

Breitmeyer, B. G.. Levi, D. M., \& Harwerth, R. S. (1981). Flicker masking in spatial vision. Vision Research, 21, 1377-1385.

BrigGs, G. G., \& KinsBourne, M. (1972). Visual persistence as measured by reaction time. Quarterly Journal of Experimental Psychologv, 24, 318-325.

Coltheart, M. (1980). Iconic memory and visible persistence. Perception \& Psychophysics, 27, 183-228.

Di LoLlo, V. (1980). Temporal integration in visual memory. Journal of Experimental Psychology: General, 109, 75-97.

Di Lollo, V., Seiffert, A. E., Burchett, G., Rabeeh, R., \& Ruman. T. A. (1997). Phosphor persistence of oscilloscopic displays: A comparison of four phosphors. Spatial Vision, 10, 353-360.

EFRON, R. (1970). Effect of stimulus duration on perceptual onset and offset latencies. Perception \& Psychophysics, 8, 231-234.

ERIKSEN, B. A., \& ERIKSEN, C. W. (1974). Effects of noise letters upon the identification of a target letter in a nonsearch task. Perception \& Psychophysics, 16, 143-149.

May, J. G., Martin, F., MacCana, F., \& Lovegrove, W. J. (1988). The effects of spatial frequency and temporal waveform on three measures of temporal processing. Journal of General Psychology, 115, 293-306.

OsGOOD, C. E. (1953). Method and theory in experimental psychology. New York: Oxford University Press.

PARKer, D. M. (1980). Simple reaction times to the onset, offset, and contrast reversal of sinusoidal grating stimuli. Perception \& Psychophysics, 28, 365-368.

Servière, J., Miceli, D., \& Galifret, Y. (1977). Electrophysiological correlates of the visual perception of "instantaneous" and "durable." Vision Research, 17, 57-63.

Smith, A. T., Early. F., \& Grogan, S. C. (1986). Flicker masking and developmental dyslexia. Perception, 15, 473-482.

(Manuscript received July 15, 1998; revision accepted for publication November 10, 1998.)

\title{
Nominations for the Editorship of Memory \& Cognition
}

Nominations are solicited for the editorship of Memory \& Cognition. The term of the present editor, Morton Ann Gernsbacher, expires at the end of 2001. The new editor will begin an official 5-year term on January 1, 2002, and will begin to receive manuscripts early in January 2001. The Publications Committee of the Psychonomic Society expects to appoint the new editor by December 2000.

Nominations (including self-nominations) should be submitted by May 1, 2000, to:

\author{
Geoffrey R. Loftus \\ Chair, Memory \& Cognition Search Committee \\ Department of Psychology, Box 351525 \\ University of Washington \\ Seattle, WA 98195
}

